\title{
Démographie de la France : la double alerte
}

Dans l'histoire démographique de la France, l'année 2015 restera-t-elle un double incident de parcours ou un vrai tournant ? Au plan quantitatif, les changements sont incontestables : nette diminution des naissances, décrochage à la baisse de la fécondité, forte hausse des décès. Une analyse approfondie suppose aussi de choisir la bonne échelle géographique.

\section{Retenir une bonne échelle d'analyse}

La compréhension des évolutions démographiques de la France en 2015 requiert, contrairement à la quasi-totalité des résultats diffusés, de s'en tenir à la France métropolitaine au lieu de considérer l'ensemble constitué par la France métropolitaine plus les 5 départements d'outremer (Guadeloupe, Guyane, Martinique, Réunion et Mayotte $)^{1}$. Certes, une telle addition reste pertinente pour connaître le nombre d'habitants de la France - même si elle est insuffisante puisque la population de la France doit inclure aussi les habitants des pays et territoires d'outre$\mathrm{mer}^{2}$. Mais chaque département d'outre-mer connait un régime démographique et un système migratoire qui lui sont propres. Que de différences, par exemple, entre la fécondité élevée de la Guyane et celle, nettement moindre, de la Guadeloupe ! Quelle évolution inverse entre le solde migratoire très élevé de Mayotte et le solde migratoire négatif de la Martinique ${ }^{3}$ ! Il importe donc de considérer comme échelle d'analyse pertinente la seule France métropolitaine, dont tous les territoires, même s'ils présentent une certaine diversité, relèvent d'un même moule historico-géographique.

\section{Politique familiale et niveau de fécondité}

La baisse du nombre de naissances en France métropolitaine en 2015 est nette : moins 19000 naissances, soit un recul de 2,5\% par rapport à l'année précédente sachant que la fécondité est passée de 1,98 enfant par femme en 2014 à 1,93 en 2015. Comment expliquer une telle baisse ? La mauvaise santé économique de la France ne paraît pas déterminante puisque la crise a commencé en 2008 sans effet baissier significatif sur la fécondité. Mais cette mauvaise santé engendre bien des effets démographiques dans un pays nettement moins attractif pour les immigrants que l'Allemagne, l'Autriche ou le Royaume-Uni, d'où, par exemple, les problèmes constatés à Calais du fait de

\footnotetext{
1. D'où les tableaux inédits $A$ et $B$, p. 19
}

2. Polynésie française, Saint-Barthélemy, Saint-Martin, Saint-Pierre-et-Miquelon, Wallis et Futuna, Nouvelle-Calédonie (ainsi que les Terres australes et antarctiques françaises et Clipperton dans le Pacifique).

3. Sur ces données différenciées, cf. Dumont, Gérard-François, « La démographie de la France, une réalité plurielle », Population \& Avenir, ${ }^{\circ} 712$, mars-avril 2013. [www.population-demographie.org/revue04.htm] la localisation géographique de la ville dans les réseaux à destination d'outre-Manche.

Or, les variations de fécondité de la France métropolitaine sont, ces dernières décennies, essentiellement ${ }^{4}$ liées à des changements dans la politique familiale et dans la confiance en cette dernière. Pour ne prendre qu'un exemple, la baisse de 1981-1982 s'explique par la remise en cause de certains aspects de cette politique, et la hausse de 1985 par l'aménagement de nouvelles prestations permettant de concilier plus facilement vie professionnelle et vie familiale. Il n'y a d'ailleurs rien d'étonnant à ce que les décisions de politique familiale aient des effets : si les décisions de politique économique ont des conséquences, celles de la politique familiale ne peuvent être neutres.

\section{Les naissances et les décès en France}

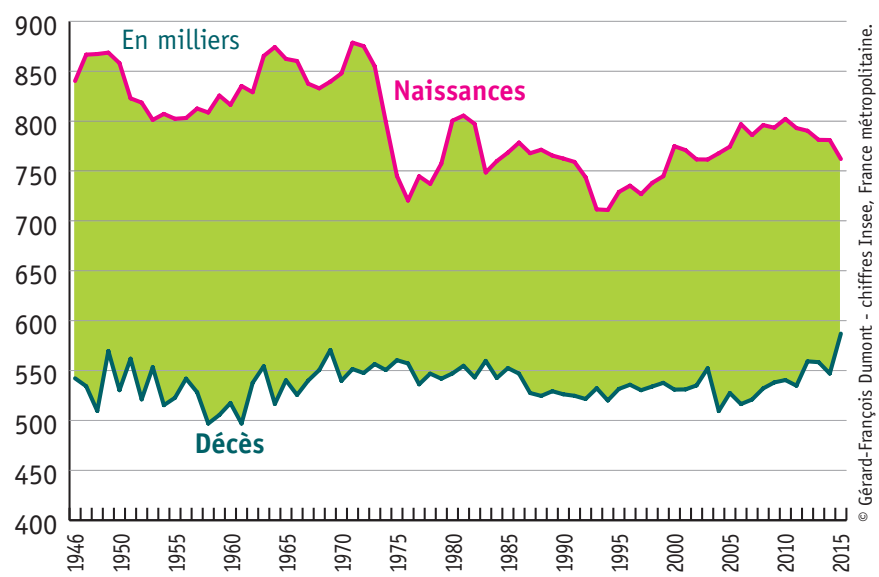

Depuis 1946, la France enregistre constamment un excédent des naissances sur les décès; ce dernier diminue avec la baisse de fécondité depuis les années 1970, tandis que les décès semblent appelés à augmenter, ne serait-ce qu'en raison du vieillissement de la population.

Or, la politique familiale a connu en France d'importants changements ces dernières années, comme la diminution du complément de mode de garde (CMG), versé pour aider les parents employant une nourrice à domicile ou une assistante maternelle, la majoration des allocations familiales reportée de deux ans ou le plafonnement fortement abaissé du quotient familial. Il faut y ajouter la mise sous condition de ressources des allocations familiales, qui engendre des effets de seuil, injustes par nature, et qui balaie trois quarts de siècle d'une politique familiale que tous les gouvernements - de droite comme de gauche - avaient pérennisée ${ }^{5}$. En outre, nombre de Français ont compris que les importantes baisses de dotations ${ }^{6}$ de l'État aux

4. D'autres facteurs peuvent s'exercer, comme cela s'est constaté en 2000

5. Sachant que la même décision prise en 1998 avait été remisée au bout de neuf mois, notamment face au mécontentement exprimé au sein de la gauche plurielle, dont celle du parti communiste.

6. Notamment avec la réduction de dotation globale de fonctionnement (DGF) de 3,5 milliards d'euros dans le budget 2016, sur un montant de plus de 36 milliards d'euros. La dotation de 2017 devrait être inférieure de 11 milliards d'euros à celle de 2014 et en retrait de 12,5 milliards par rapport à 2013. 
2. La fécondité de la France métropolitaine ET LE NIVEAU DE REMPLACEMENT

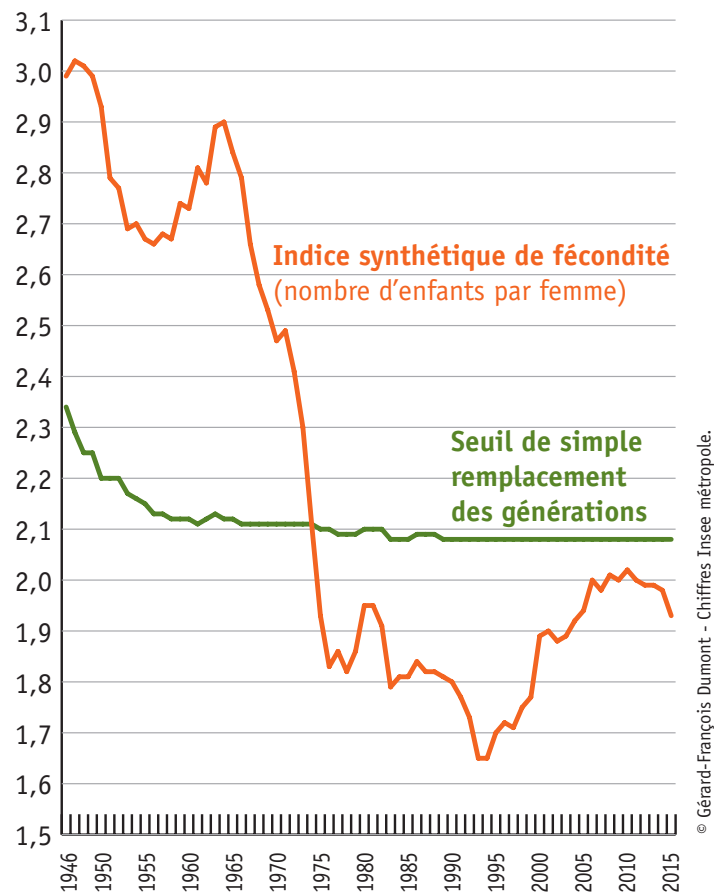

D) collectivités territoriales vont entraîner la suppression ou la réduction des projets de nouvelles crèches ou de relais d'assistantes maternelles.

Cela laisse penser que, face à la moindre compensation des charges familiales et à davantage de difficultés pour concilier vie professionnelle et vie familiale, la population française a arbitré défavorablement en ce qui concerne l'accueil de l'enfant. Est-ce le début d'une perte durable de confiance dans une politique familiale beaucoup moins solidaire ? L'avenir le dira.

\section{Une baisse historique de l'espérance de vie ?}

La seconde alerte de l'année 2015 tient à la forte hausse des décès : plus 40000 en France métropolitaine, soit une hausse supérieure à $7 \%$. A priori, les causes sont bien renseignées : grippe sévère, vaccin antigrippe peu efficace, canicule modeste mais réelle. Notons toutefois que l'année 2015 enregistre la seule baisse de l'espérance de vie depuis la forte canicule de 2003, qui avait causé 15000 décès supplémentaires ${ }^{7}$. Et d'autres années ont enregistré des surcroîts de décès dus à des hivers plus mortifères sans engendrer de baisse de l'espérance de vie.

Le mauvais résultat de la mortalité en $2015^{8}$ est l'occasion de rappeler que le haut niveau d'espérance de vie atteint au début des années 2010, suite à une progression assez

7. Dumont, Gérard-François, Montenay, Yves, « Le dernier bilan de la canicule : un pic historique et une géographie précise », Population \& Avenir, $n^{\circ} 668$, mai-juin 2004. [www.population-demographie.org/revue04c.htm]

8. Sur une plus longue période, cf. Dumont, Gérard-François, « La longévité en France : un bilan dual », Population \& Avenir, $n^{\circ} 722$, mars-avril 2015. [www.population-demographie.org/revue04.htm]

régulière au fil des décennies, et d'ailleurs généralement imprévue, n'est pas un acquis. L'évolution de l'espérance de vie dépend de facteurs plutôt externes aux individus, comme l'efficacité du système sanitaire ou les innovations médicales et pharmaceutiques, mais aussi de leurs comportements. Elle dépend donc à la fois de la façon de vivre des personnes et, par exemple, des contrôles sur l'utilisation des pesticides.

Derrière la forte hausse de décès en 2015, due il est vrai en partie au vieillissement de la population, ne pourrionsnous pas aussi déceler les possibles effets de la consommation de produits alimentaires dont la composition finit, au fil des années, par affecter la santé des consommateurs, certains comportements alimentaires se traduisant par la hausse des taux d'obésité, des moyens insuffisants pour se soigner caractérisant une partie de plus en plus importante de notre population, ou encore une offre médicale lacunaire dans certains territoires ? Si la réponse à cette question était positive, la baisse de l'espérance de vie en 2015 serait ce qu'on appelle en prospective un « signal faible ", c'est-à-dire annonciateur d'une évolution de l'espérance de vie moins favorable dans le futur que dans les décennies précédentes.

3. EvOLUTION DEPUIS 1995 DE L'INDICE MENSUEL de fÉcondité en France métropolitaine

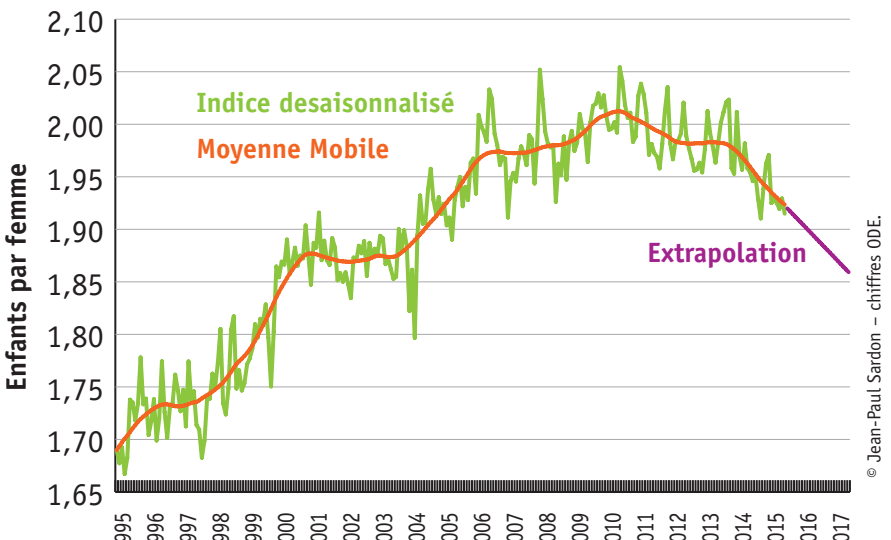

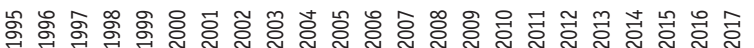

\section{L'éclairage de l'évolution des naissances par celle de la baisse mensuelle de la fécondité}

La baisse de 16000 naissances enregistrée au cours des 9 premiers mois de 2015, par rapport à la même période de 2014, amplifiée par une nouvelle baisse en octobre, et maintenant confirmée pour l'année entière, montrait que la diminution du nombre de femmes en âge de procréer ne peut à elle seule l'expliquer. Et ce d'autant plus que le nombre de femmes en âge d'avoir des enfants recule depuis 1993, ce qui n'avait pas empêché le nombre de naissances d'augmenter entre 2004 et 2010.
L'indicateur de fécondité de la France métropolitaine s'élève à 1,931 enfant par femme en 2015 contre 1,977 en 2014.

C'est en 2010 que l'indicateur de fécondité a atteint un maximum de 2,015, soit sa valeur la plus élevée depuis 1975. Depuis, il baissait légèrement, mais cette baisse s'est nettement amplifiée en 2015, avec un recul plus élevé au cours de la seule année 2015 que celui enregistré les cinq années précédentes, entre 2010 et 2014.

J.-P. S. 


\section{Le mouvement de la population de la France en quelques chiffres}

\section{A. France métropolitaine}

\begin{tabular}{|c|c|c|c|c|c|c|c|c|}
\hline \multirow{2}{*}{ Année } & \multirow{2}{*}{$\begin{array}{c}\text { Population } \\
\text { moyenne }\end{array}$} & \multicolumn{4}{|c|}{ Chiffres en milliers } & \multicolumn{3}{|c|}{ Taux pour 1000 habitants } \\
\hline & & Naissances vivantes & Décès & Solde naturel & Solde migratoire évalué & Natalité & Mortalité & Accroissement naturel \\
\hline 1980 & 53880 & 800,4 & 547,1 & $+253,3$ & +44 & 14,9 & 10,2 & $+4,7$ \\
\hline 1985 & 55284 & 768,4 & 552,5 & $+215,9$ & +38 & 13,9 & 10,0 & $+3,9$ \\
\hline 1990 & 56708 & 762,4 & 526,2 & $+236,2$ & +80 & 13,4 & 9,3 & $+4,1$ \\
\hline 1995 & 57844 & 729,6 & 531,6 & $+198,0$ & +40 & 12,6 & 9,2 & $+3,4$ \\
\hline 1999 & 58677 & 744,8 & 537,7 & $+207,1$ & +60 & 12,7 & 9,2 & $+3,5$ \\
\hline 2000 & 59062 & 774,8 & 530,9 & $+243,9$ & +70 & 13,1 & 9,0 & $+4,1$ \\
\hline 2001 & 59476 & 770,9 & 531,1 & $+239,8$ & +85 & 13,0 & 8,9 & $+4,1$ \\
\hline 2002 & 59894 & 761,6 & 535,1 & $+226,5$ & +95 & 12,7 & 8,9 & $+3,8$ \\
\hline 2003 & 60304 & 761,5 & 552,3 & $+209,2$ & +100 & 12,6 & 9,2 & $+3,4$ \\
\hline 2004 & 60734 & 767,8 & 509,4 & $+258,4$ & +105 & 12,7 & 8,4 & $+4,3$ \\
\hline 2005 & 61181 & 774,4 & 527,5 & $+246,9$ & +95 & 12,7 & 8,6 & $+4,1$ \\
\hline 2006 & 61597 & 796,9 & 516,4 & $+280,5$ & +115 & 12,9 & 8,4 & $+4,5$ \\
\hline 2007 & 61965 & 786,0 & 521,0 & $+265,0$ & +75 & 12,7 & 8,4 & $+4,3$ \\
\hline 2008 & 62304 & 796,0 & 532,1 & $+263,9$ & +67 & 12,8 & 8,5 & $+4,3$ \\
\hline 2009 & 62619 & 793,4 & 538,1 & $+255,3$ & +44 & 12,7 & 8,6 & $+4,1$ \\
\hline 2010 & 62917 & 802,2 & 540,5 & $+261,7$ & +43 & 12,8 & 8,6 & $+4,2$ \\
\hline 2011 & 63223 & 793,0 & 534,8 & $\begin{array}{r}+258,2 \\
\end{array}$ & +47 & 12,5 & 8,5 & $+4,0$ \\
\hline 2012 & 63537 & 790,3 & 559,2 & $+231,1$ & +91 & 12,4 & 8,8 & $+3,6$ \\
\hline $2013(p)$ & 63840 & 781,2 & 558,4 & $+222,8$ & +61 & 12,2 & 8,8 & $+3,4$ \\
\hline $2014(p)$ & 64129 & 781,0 & 547,0 & $+234,0$ & +61 & 12,2 & 8,5 & $+3,7$ \\
\hline 2015 (p) & 64395 & 762,0 & 587,0 & $+175,0$ & +61 & 11,8 & 9,1 & $+2,7$ \\
\hline
\end{tabular}

B. France métropolitaine + Dom (Guadeloupe*, Guyane, Martinique, La Réunion)

\begin{tabular}{|c|c|c|c|c|c|c|c|c|}
\hline \multirow{2}{*}{ Année } & \multirow{2}{*}{$\begin{array}{l}\text { Population } \\
\text { moyenne }\end{array}$} & \multicolumn{4}{|c|}{ Chiffres en milliers } & \multicolumn{3}{|c|}{ Taux pour 1000 habitants } \\
\hline & & Naissances vivantes & Décès & Solde naturel & Solde migratoire évalué & Natalité & Mortalité & Accroissement naturel \\
\hline 1999 & 60316 & 775,8 & 547,3 & $+228,5$ & +61 & 12,9 & 9,1 & $+3,8$ \\
\hline 2000 & 60725 & 807,4 & 540,6 & $+266,8$ & +71 & 13,3 & 8,9 & $+4,4$ \\
\hline 2001 & 61163 & 803,2 & 541,0 & $+262,2$ & +87 & 13,1 & 8,8 & $+4,3$ \\
\hline 2002 & 61605 & 792,7 & 545,2 & $+247,5$ & +97 & 12,9 & 8,9 & $+4,0$ \\
\hline 2003 & 62038 & 793,0 & 562,5 & $+230,5$ & +102 & 12,8 & 9,1 & $+3,7$ \\
\hline 2004 & 62491 & 799,4 & 519,5 & $+279,9$ & +105 & 12,8 & 8,3 & $+4,5$ \\
\hline 2005 & 62959 & 806,8 & 538,1 & $+268,7$ & +92 & 12,9 & 8,5 & $+4,4$ \\
\hline 2006 & 63394 & 829,3 & 526,9 & $+302,4$ & +112 & 13,1 & 8,3 & $+4,8$ \\
\hline 2007 & 63782 & 818,7 & 531,2 & $+287,5$ & +74 & 12,8 & 8,3 & $+4,5$ \\
\hline 2008 & 64134 & 828,4 & 542,6 & $+285,8$ & +57 & 12,9 & 8,5 & $+4,4$ \\
\hline 2009 & 64459 & 824,6 & 548,5 & $+276,1$ & +32 & 12,8 & 8,5 & $+4,3$ \\
\hline 2010 & 64773 & 832,8 & 551,2 & $+281,6$ & +39 & 12,9 & 8,5 & $+4,4$ \\
\hline 2011 & 65087 & 823,4 & 545,1 & $+278,3$ & +30 & 12,6 & 8,4 & $+4,2$ \\
\hline 2012 & 65403 & 821,0 & 569,9 & $+251,1$ & +72 & 12,6 & 8,7 & $+3,9$ \\
\hline 2013 & 65710 & 811,5 & 569,2 & $+242,3$ & +47 & 12,3 & 8,7 & $+3,6$ \\
\hline $2014(p)$ & 66117 & 811,4 & 558,7 & $+252,7$ & +47 & 12,3 & 8,5 & $+3,8$ \\
\hline \multicolumn{9}{|c|}{ À partir de 2014, y compris Mayotte. } \\
\hline $2014(p)$ & 66227 & 818,6 & 559,3 & $+259,3$ & +47 & 12,4 & 8,4 & $+4,0$ \\
\hline $2015(p)$ & 66504 & 800,0 & 600,0 & $+200,0$ & +47 & 12,0 & 9,0 & $+3,0$ \\
\hline
\end{tabular}

\section{CLIN D'CEIL}

\section{SOLUTION DE LA PAGE 2}

Tristan da Cunha est à la fois le nom d'un archipel et de l'île principale de cet archipel qui compte $96 \mathrm{~km}^{2}$ de superficie. Lî̂le commence à apparaître sur les cartes à partir de 1509 et sert de point de repère et de point de ravitaillement en eau douce sur la route des Indes.

Quant au peuplement, on peut dire qu'elle ne sera vraiment colonisée qu'à partir de 1810 .. par un excentrique natif du Massachusetts, un certain Jonathan Lambert, qui prend possession des terres en son nom propre et les rebaptise Refreshment Islands. Et, enfin, c'est à cause de... Napoléon que les Britanniques, en 1816, finissent par l'annexer pour empêcher toute tentative d'évasion de l'empereur détenu sur Sainte-Hélène.

C'est le lieu habité (267 personnes) le plus isolé du monde, puisque l'île volcanique de Tristan da Cunha est située dans l'Atlantique Sud, à 2807 km de l'Afrique du Sud et à $3360 \mathrm{~km}$ de l'Amérique du Sud. Elle est rattachée administrativement à l'archipel britannique de Sainte-Hélène, qui est tout de même à $2418 \mathrm{~km}$ de distance !
Un bateau ravitaille l'île tous les mois et demi... En 1961, il avait fallu évacuer toute la population vers le Royaume-Uni à cause de l'éruption du volcan Queen Mary. En 1963, lorsqu'il fut constaté que le volcan n'avait pas tout détruit, les Tristanais ont tenu à regagner leur île, préférant tous, sauf cinq, « vivre et travailler au pays », cinq autres ayant décédé lors de leur séjour en Angleterre. Mais la population s'était accrue de huit nouveau-nés entre temps... 